PANCREATIC CANCER

\title{
KRAS dosage key in PDAC
}

Oncogenic gene dosage is a key factor in pancreatic adenocarcinoma (PDAC), according to new research.

Work to associate the mutational landscape of PDAC with tumour phenotypes has been largely unsuccessful. Factors hindering these studies include the complexity of PDAC genomes, high stromal content (impairing gene-dosage and transcriptome analyses) and a lack of cell culture resources.

To address these limitations, Roland Rad and colleagues derived cancer cell cultures from untreated mouse PDAC tumours, driven by pancreatic Kras ${ }^{G 12 D}$ mutation. These cell cultures were characterized on the basis of genomic, transcriptomic and phenotypic features.
The first key finding was that two-thirds of cancers had increased $\mathrm{Kras}^{\mathrm{G} 12 \mathrm{D}}$ gene dosage (termed $\mathrm{Kras}^{\mathrm{i} D}$ ). By analysing human pancreatic intraepithelial neoplasias, the researchers showed that, after initial KRAS mutation, additional oncogenic gene dosage gain is required for cancer evolution, via amplification of either mutated Kras or other oncogenes, such as Yap1 or Nfkb2. Kras ${ }^{i G D}$ was also shown to promote metastasis, providing a mechanism for the frequent early metastasis of PDAC in humans.

Next, using a variety of mouse models, Rad and colleagues demonstrated that the nature of oncogenic gene dosage gain is dependent on the inactivated tumour suppressor. For instance, homozygotic inactivation of Tp53 or
Cdkn2a predisposed tumours to Kras ${ }^{i G D}$; conversely, Yap1 or Nfkb2 amplifications resulted from heterozygotic C $d k n 2 a$ loss.

Finally, integration of cellular phenotypes showed that Kras mutational status and gene dosage was associated with epithelial-tomesenchymal transition and the degree of dedifferentiation. "The current progression model considers the KRAS mutations, but not its dosage variation," concludes Rad. "We have introduced oncogenic dosage increase as a fundamentally important process." Hugh Thomas

ORIGINAL ARTICLE Mueller, S. et al. Evolutionary routes and KRAS dosage define pancreatic cancer phenotypes. Nature http://dx.doi.org/10.1038/ nature25459 (2018) 